\title{
CRIMSONpublishers
}

http://www.crimsonpublishers.com

\section{Patient Centered Outcome Assessment for Periodontal Therapy}

\author{
Amit Arvind Agrawal* \\ Department of Periodontics, MGV's KBH Dental College and Hospital, India
}

*Corresponding author: Amit Arvind Agrawal, Professor and PG guide, Department of Periodontics, MGV's KBH Dental College and Hospital, Nasik, Maharashtra, India

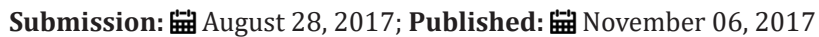

\begin{abstract}
Patient centered outcomes research means the investigation that leads to clarity about outcomes that matter to patients and how to efficiently achieve those outcomes. In periodontics, symptoms such as redness, bleeding on brushing, loosening of affected teeth and persistent bad breath are highly relevant from the patients point of view and often have a considerable adverse impact on their daily quality of life. Including these parameters in treatment plan and in clinical research will result in better patient compliance and quality of care that matters to the patient.
\end{abstract}

A range of measures have been developed and validated to evaluate oral health related quality of life (OHRQoL) all of which are similar in their ability to detect changes in the physical, functional and psychosocial impacts of oral disorders and hence suitable for use in clinical studies. Healthcare is no longer a disease-centric process. Modern healthcare is patient centric, where patients (and their families) are active participants in care.

Keywords: Oral health related quality of life; Patient-centered outcome; Clinical outcome assessment; True end points

\section{Introduction}

A clinical outcome assessment (COA) measures patient symptoms, mental state or the effects of a disease on patient functions. A COA can be used to determine whether or not a drug/ treatment/procedure has been demonstrated to provide treatment benefit (i.e. a benefit compared with other drug/treatments). There are 5 types of COA:

1. Patient-reported outcomes (PRO).

2. Clinician reported outcomes (ClinRO).

3. Oberserver-reported outcomes (ObsRO).

4. Performance outcomes (PerfO).

5. Biomarkers.

Patient-centered care: "is respectful of and responsive to individual patient preferences, needs, and values [and those of family and friends] and ensures that patient's values guide all clinical decisions". Patient-based outcomes (PBOs) also called as "true endpoints" are subjective measures which capture patients' perspectives of disease or therapy and complement conventional clinical (surrogate) measures.

'Patient-centered outcomes research' means the investigation that leads to clarity about outcomes that matter to patients and how to efficiently achieve those outcomes and is an important discussion catalyst for patients and their clinicians. Thus, bedside caregivers must be knowledgeable of the findings of that research. Correspondingly, they must be prepared to use that knowledge to assist patients and families in making informed healthcare decisions. Clinicians are expected to implement the results of outcomes research to help patients and families identify care and treatment preferences. Despite the emphasis on patient-centered care, medical decisions do not always reflect a high level of collaboration with patients and their families. Current practice may not yet be meeting the challenge of ensuring that patient-centered care is consistently implemented.

Improving the patient experience of care is a component of the "Triple Aim" ("Better Health, Better Care and Lower Cost") of healthcare [1]. Patient-centered processes of care embrace the idea that patients (and their families) know themselves best and that optimal outcomes arise from integration of patients and families into those processes. For example, patient participation in medical decision making has been associated with improved patient satisfaction and, perhaps more importantly, with outcomes [2].

\section{Periodontal Perspective}

Traditionally, periodontal disease has been defined and measured using surrogate markers, most commonly-pocket probing depth (PPD) and clinical attachment loss (CAL). However, PBOs are 
reported to be more relevant to patient's daily lives than objective changes in PPD. In periodontics, the condition of oral cavity affects the ability to eat, speak, and socialize as well as interpersonal relationships and daily activities; these may be due to symptoms such as redness, bleeding on brushing, loosening of affected teeth, and persistent bad breath. These symptoms are not normally documented in a research report. Such symptoms, however, are highly relevant from the patients' point of view and often have a considerable adverse impact on their daily quality of life (QoL). Patient-based outcomes were identified as a research priority at the 2003 World Workshop on Emerging Science in Periodontology [3]. Assessment of PBOs is important in periodontal therapy as patients' opinions may differ from traditional clinical endpoints $\mathrm{Ng} \&$ Leung [4]. Oral health-related quality of life (OHRQoL) is one PBO and is recognized as an integral part of general health and well-being WHO, John et al. [5]. A range of measures have been developed and validated to evaluate OHRQoL, all of which are similar in their ability to detect changes in the physical, functional and psychosocial impacts of oral disorders and hence suitable for use in clinical studies [6-8].

\section{Patients Perception of Own Efforts}

Vatne et al. [9] did a study to investigate patients' response to systematic periodontal information, motivation and treatment strategy by comparing the patients' perception of own efforts and results with those clinically registered in a trans-sectional, observational study. They concluded that periodontal treatment, including customized information and education on the etiology and pathogenesis, prevention and treatment as well as maintenance of periodontal diseases, resulted in a high degree of short- and long term compliance and very good patient centered outcomes, which again had a positive impact on the patient's satisfaction. The patient centered outcomes correlated mostly with the compared clinical endpoints. This also strengthens the point stated by Wilson [10] that informing patients adequately causes increased compliance and reduced anxiety towards prospective therapy, emphasizing the need to assess these patient-related factors.

\section{Surgical vs Non-Surgical Approach}

The results of a systematic review of 11 studies by Shanbhag et al. [11] suggests that all forms of non-surgical periodontal therapy can improve the OHRQoL of adult patients with periodontal disease in the immediate (1 week) and long-term (12 months). The OHRQoL domains that improve after periodontal therapy are those of function, psychology and pain. Surgical therapy does not have a significant additional benefit on the OHRQoL of patients who have already received non-surgical therapy. When provided alone, surgical therapy may be associated with more negative impacts, but can improve OHRQoL when provided with EMD (Enamel Matrix Derivative), 1 week post-treatment. Table 1 [12-18] shows the frequency of different Oral Health-related Quality of Life (OHRQoL) measures used in the included studies [11]. Other than these; there is a WHO-QOL-BREF measure to assess the frequency, intensity, capability, and evaluation of the performed actions [18].

\section{Surgical Therapy}

Recently Chou et al. [19] conducted a study to compare the difference in QoL in patients treated with regenerative (RG) and respective surgery (RS). Results indicated that both RG and RS can improve patients QoL, particularly regarding physical pain and psychological discomfort. Following periodontal treatment, the RG patients obtained more favorable QoL scores (i.e. lower scores) than the RS patients did. Compared with the patients who underwent RS, the functional limitation of patients who underwent RG improved significantly more.

\section{Soft Tissue Augmentation Procedures}

McGuire et al. [20] reviewed patient-reported outcome (PRO) metrics for dentistry, and in particular, periodontics. The PRO commentary for periodontics includes a review of split mouth, randomized, controlled clinical trial results that specifically tracked pain at different sites over time after intervention and provided guidelines for peak pain time points and evidence for referred pain assessment when studying soft tissue augmentation procedures. Both the questions that are asked of patients and the timing of those questions are important study design considerations. The authors suggested PRO methodology for periodontal clinical trials that can be used to identify information important to patients and clinicians [21].

\section{Implantology}

Patient-centered outcome measures in a study by Vercruyssen et al. [22] were the Dutch version of the McGill Pain Questionnaire (MPQ-DLV), the health-related quality of life instrument (HRQOL), visual analog scales (VAS), the duration of the procedure, and the analgesic doses taken each day. Within the limitations of the study, no difference was found in patient-centered outcome variables after immediate or delayed loading of dental implants.

\section{Patient Centered Outcomes Research Institute (PCORI)}

The still new 'PCORI' [12-15] is changing the world of clinical research by bringing the voice of patients and other stakeholders into the design, conduct and interpretation of research. PCORI is both championing and riding the wave of movements that combine rigor and relevance, carefulness and openness, expertise and inclusive participation. These movements challenge entrenched oligarchies. Now PCORI is seeking to add to the process of timely, accessible, transparent communication of research results to increase the credibility of patient-centered outcomes research [16,17-28].

\section{Conclusion}

Healthcare is no longer a disease-centric process. Modern healthcare is patient-centric, where patients (and their families) are active participants in care.

The value of patient-centered outcomes research lies in insights from patients and their families that can be used to help guide clinical care and ensure that care needs and goals are met. The 
patient-centric perspective is essential in promoting optimal care. This is most important in critical care where the nature of critical illness often entails uncertainty about prognosis and outcomes.

Patient-centered outcomes research also stimulates further investigation to improve the critical care hospitalization experience, as applicable. By assessing the impact of interventions, such as family presence, open visitation, family care conferences, and patient and family engagement on committees and in educational initiatives, improvements in critical care and best practices will surely follow.

The future of patient-centered outcomes research holds much potential. As clinicians, we must stay abreast of the research, assess its utility in our patients, and integrate the important advances into our care plans and processes.

\section{References}

1. Berwick DM, Nolan TW, Whittington J (2008) The triple aim: care, health, and cost. Health Aff (Millwood) 27(3): 759-769.

2. Tak HJ, Ruhnke GW, Meltzer DO (2013) Association of patient preferences for participation in decision making with length of stay and costs among hospitalized patients. JAMA Intern Med 173(13): 1195-1203.

3. Hujoel PP (2004) Endpoints in periodontal trials: the need for an evidence-based research approach. Periodontol 2000 36: 196-204.

4. Leung WK, Ng SKS (2006) Oral health-related quality of life and periodontal status. Community Dent Oral Epidemiol 34(2): 114-122.

5. John MT, Slade GD, Szentpetery AS, Jurgen M (2004) Oral health-related quality of life in patients treated with fixed, removable, and complete dentures 1 month and 6-12 months after treatment. Int J Prostho 17(5): 503-511.

6. Slade GD (1997) Derivation and validation of a short-form oral health impact profile. Community Dentistry and Oral Epidemiol 25(4): 284290.

7. Locker D, Allen F (2007) What do measures of 'oral health-related quality of life' measure. Community Dentistry and Oral Epidemiol 35(6): 401-411.

8. Sischo L, Broder HL (2011) Oral health-related quality of life: what, why, how, and future implications. J Dent Res 90(11): 1264-1270.

9. Vatne JF, Gjermo P, Sandvik L, Preus HR (2015) Patients perception of own efforts versus clinically observed outcomes of non-surgical periodontal therapy in a Norwegian population: an observational study. BMC Oral Health 15: 61.

10. Wilson TG (1996) Compliance and its role in periodontal therapy. Periodontol 2000 12: 16-23

11. Shanbhag S, Dahiya M, Croucher R (2012) The impact of periodontal therapy on oral health-related quality of life in adults: a systematic review. J Clin Periodontol 39(8): 725-735.

12. Selby JV, Beal AC, Frank L (2012) The Patient-Centered Outcomes Research Institute (PCORI) national priorities for research and initial research agenda. JAMA 307(15): 1583-1584.
13. Ozcelik O, Haytac MC, Seydaoglu G (2007) Immediate post-operative effects of different periodontal treatment modalities on oral healthrelated quality of life: a randomized clinical trial. Jr Clin Periodontol 34(9): 788-796.

14. Bajwa A, Watts TL, Newton JT (2007) Health control beliefs and quality of life con-side rations before and during periodontal treatment. Oral Health Prev Dent 5(2): 101-104.

15. Jowett AK, Orr MT, Rawlinson A, Robinson PG (2009) Psychosocial impact of periodontal disease and its treatment with 24-h root surface debridement. Jr Clin Periodontol 36(5): 413-418.

16. Ohrn K, Jonsson B (2012) A comparison of two questionnaires measuring oral health-related quality of life before and after dental hygiene treatment in patients with periodontal disease. Int J Dent Hyg 10(1): 9-14.

17. Wong RM, Ng SK, Corbet EF, Keung Leung W (2012) Non-surgical periodontal therapy improves oral health-related quality of life. J Clin Periodontol 39(1): 53-61.

18. WHOQOL-BREF introduction, administration, scoring and generic version of the assessment, field trial version December 1996. Programme on Mental Health, World Health Organization Geneva, Switzerland.

19. Chou YH, Yang YH, Kuo HC, Ho KY, Wang WC, et al. (2017) Periodontal surgery improves oral health-related quality of life in chronic periodontitis patients in Asian population. Kaohsiung J Med Sci 33(10): 523-529.

20. McGuire MK, Scheyer ET, Gwaltney C (2014) Commentary: incorporating patient-reported outcomes in periodontal clinical trials. J Periodontol 85(10): 1313-1319

21. Vercruyssen M, Cox C, Naert I, Jacobs R, Teughels W, et al. (2015) Accuracy and patient-centered outcome variables in guided implant surgery: A RCT comparing immediate with delayed loading. Clin Oral Implants Res, pp. 1-6.

22. https://www.pcori.org/research-results/patient-centered-outcomesresearch.

23. Saito A, Hosaka Y, Kikuchi M (2010) Effect of initial periodontal therapy on oral health-related quality of life in patients with periodontitis in Japan. J Periodontol 81(7): 1001-1009.

24. Saito A, Ota K, Hosaka Y (2011) Potential impact of surgical periodontal therapy on oral health-related quality of life in patients with periodontitis: a pilot study. J Clin Periodontol 38(12): 1115-1121.

25. Aslund M, Suvan J, Moles DR, D'Aiuto F, Tonetti MS (2008) Effects of two different methods of non-surgical periodontal ther- apy on patient perception of pain and quality of life: a randomized controlled clinical trial. J Periodontol 79(6): 1031-1040.

26. Nagarajan S, Chandra RV (2012) Perception of oral health related quality of life (OHQoL-UK) among periodontal risk patients before and after periodontal therapy. Community Dent Health 29(1): 90-94.

27. Tsakos G, Bernabe' E, D’Aiuto F, Pikhart H, Tonetti M, et al. (2010) Assessing the minimally important difference in the oral impact on daily performances index in patients treated for periodontitis. J Clin Periodontol 37(10): 903-909.

28. Pereira LJ, Gazolla CM, Magalhães IB, Ramos Jorge ML, Marques LS, et al. (2011) Treatment of chronic periodontitis and its impact on mastication. Journal of Periodontology 82(2): 243-250. 